BULLETIN OF THE

AMERICAN MATHEMATICAL SOCIETY

Volume 79, Number 4, July 1973

\title{
MAPPINGS INTO HYPERBOLIC SPACES
}

\author{
BY MYUNG H. KWACK
}

Communicated by S. S. Chern, December 18, 1972

In this note we state some results on extensions of holomorphic mapings into hyperbolic spaces. A theorem involves extending holomorphic mappings to a domain of holomorphy. An extension problem of holomorphic mappings into a taut complex space was considered by Fujimoto [1].

Another result is that the space of all meromorphic mappings from a complex space $X$ into a hyperbolically imbedded space in $Y$ is relatively compact in the space of all meromorphic mappings from $X$ into $Y$.

A relatively compact complex space $M$ is said to be hyperbolically imbedded in a complex space $Y$ if for all sequences $\left\{p_{n}\right\}$ and $\left\{q_{n}\right\}$ in $M$ such that $p_{n} \rightarrow p \in \bar{M}$ and $q_{n} \rightarrow q \in \bar{M}$ and such that $d_{M}\left(p_{n}, q_{n}\right) \rightarrow 0$, we have $p=q$. Here $d_{M}$ denotes the pseudo-distance defined by Kobayashi [5]. A relatively compact complex space $M$ in $Y$ is strictly Levi pseudoconvex if for every point $p \in \partial M$ there are a neighborhood $U_{p}$ of $p$ and a biholomorphic map $\Phi_{p}$ of $U_{p}$ onto a subvariety of a domain $D_{p}$ in some $C^{n}$ and a function $\varphi$ defined in $U_{p}$ such that $\varphi \circ \Phi_{p}^{-1}$ is the restriction to $\Phi_{p}\left(U_{p}\right)$ of a strictly pluri-subharmonic function $\tilde{\varphi}_{p}$ defined in $D_{p}$ and $\Phi_{p}\left(U_{p} \cap M\right)=\left\{x \in \Phi\left(U_{p}\right): \tilde{\varphi}_{p}(x)<0\right\}$.

TheOREM 1. Let $X$ be a complex manifold and $A$ be an analytic subset of $X$ of codimension at least 1 . Let $M$ be a strictly Levi pseudoconvex hyperbolic space in $Y$. Then a holomorphic mapping $f$ of $X-A$ into $M$ can be extended holomorphically to a mapping $\tilde{f}$ of $X$ into $M$.

This theorem can be proved using a theorem by Kwack [6] and the fact that there exist a neighborhood $W$ of $\partial M$ and a pluri-subharmonic function $\psi$ defined on $W$ such that $W \cap M=\{x \in M: \psi(x)<0\}$.

THEOREM 2. Let $M$ be one of the following: (i) $M$ is a hyperbolic and strictly Levi pseudoconvex subspace of a complex space $Y$, and (ii) $M$ is a complex manifold having a complete Hermitian metric $d s_{M}^{2}$ all of whose holomorphic sectional curvatures are nonpositive. Let $N$ be an (unramified) Riemann domain over a Stein manifold and $f$ be a holomorphic mapping of $N$ into $M$. Then the existence domain of the mapping $f$ from $N$ into $M$ is a Stein manifold.

AMS (MOS) subject classifications (1970). Primary 32A10, 32H20.

Key words and phrases. Holomorphic functions, hyperbolic spaces, extension of holomorphic mappings. 
Corollary. Let $f, N, M$, and $Y$ be as above and $H(N)$ be the envelope of holomorphy. Then $f: N \rightarrow M$ can be extended to a holomorphic mapping $\tilde{f}$ from $H(N)$ into $M$.

Theorem 2 was proved when $M$ is a taut complex space by Fujimoto [1]. The proof of Theorem 2 uses the following lemma and arguments used by Fujimoto.

LEMMA 1. Let $p$ be a point in $\boldsymbol{C}^{n}, n \geqq 2$. Consider hyperspheres $B$ with the center $p$ and $S$ whose boundary contains $p$. Let $D$ be the intersection of the interior of $B$ and the exterior of $S$. Then every holomorphic mapping $f$ of $D$ into $M$ has a holomorphic extension to a neighborhood of $p$ where $M$ is as in Theorem 2.

This lemma is essentially proved by Griffiths [2] when $M$ is a complex manifold having a complete Hermitian metric all of whose holomorphic sectional curvatures are nonpositive.

We can also prove

THEOREM 3. Let $X$ be a complex space $Y$ with the following property; every holomorphic mapping of the punctured disk in $C$ into $X$ extends holomorphically to a mapping of the whole disk into $X$. Then if $f: M \rightarrow X$ is a holomorphic mapping of an (unramified) Riemann domain $M$ over a Stein manifold, the existence domain of $f$ is a Stein manifold and $f: M \rightarrow X$ can be extended to a holomorphic mapping $\tilde{f}: H(M) \rightarrow X$ where $H(M)$ denotes the domain of holomorphy of $M$.

Next we state a convergence theorem for a sequence of meromorphic mappings. (See [7] for definition.)

THEOREM 4. Let $X$ be a complex space and $\left\{f_{n}\right\}$ be a sequence of meromorphic mappings of $X$ into a hyperbolically imbedded space in $Y$. Then there is a subsequence which converges uniformly on compact subsets to a meromorphic mapping $f: X \rightarrow Y$.

If $X$ is a complex manifold without singularities, then it is known that any meromorphic mapping $f$ of $X$ into a hyperbolic space is holomorphic and in this case Theorem 4 follows from theorems by Kiernan [3].

The proof of Theorem 4 uses the following theorem of Bishop [8].

THEOREM (BISHOP). The limit of a sequence of purely $k$-dimensional analytic varieties whose $2 k$-volumes are uniformly bounded is again a purely $k$-dimensional variety.

We also use the following theorem by Kiernan [4].

THEOREM (KIERNAN). $M$ is hyperbolically imbedded in $Y$ if and only if 
for each Hermitian metric $h$ on $Y$, there exists a constant $c>0$ such that $f^{*}(c h) \leqq d s_{D}^{2}$ for every holomorphic mapping of a unit disk $D$ in $C$ into $M$.

Theorem 4 may be proved using a resolution of a complex space as used by Kiernan.

\section{REFERENCES}

1. H. Fujimoto, On holomorphic maps into a taut complex space, Nagoya Math. J. 46 (1972), 49-61.

2. P. Griffiths, Two theorems on extensions of holomorphic mappings, Invent. Math. 14 (1971), 27-62.

3. P. Kiernan, Extensions of holomorphic maps, Trans. Amer. Math. Soc. (to appear).

4. - Hyperbolically imbedded spaces and the big Picard theorem, Math. Ann. (to appear).

5. S. Kobayashi, Hyperbolic manifolds and holomorphic mappings, Pure and Applied Math., 2. Marcel Dekker, Inc., 1970. MR 43 \# 3503.

6. M. Kwack, Generalization of the big Picard theorem, Ann. of Math. (2) 90 (1969), 9-22. MR 39 \# 4445.

7. R. Remmert, Holomorphe und meromorphe abbildungen komplexes raüme, Math. of Ann. 133 (1957), 328-370. MR 19, 1193.

8. G. Stolzenberg, Volumes, limits, and extensions of analytic varieties, Lecture Notes No. 19, Berlin-Heidelberg-New York, Springer, 1966. MR 34 \#6156.

Department of Mathematics, Howard University, Washington, D.C. 20001 